\title{
A NOVEL GUARANTEED COST CONTROL FOR HOPFIELD NEURAL NETWORKS WITH MULTIPLE TIME-VARYING DELAYS
}

\author{
Grienggrai Rajchakit \\ Department of Mathematics \\ Maejo University \\ Chiangmai, 50290, THAILAND
}

\begin{abstract}
This paper studies the problem of guaranteed cost control for a class of Hopfield delayed neural networks with multiple time-varying delays. The time delay is a continuous function belonging to a given interval, but not necessary to be differentiable. A cost function is considered as a nonlinear performance measure for the closed-loop system. The stabilizing controllers to be designed must satisfy some exponential stability constraints on the closed-loop poles. By constructing a set of augmented Lyapunov-Krasovskii functionals combined with Newton-Leibniz formula, a guaranteed cost controller is designed via memoryless state feedback control and new sufficient conditions for the existence of the guaranteed cost state-feedback for the system are given in terms of linear matrix inequalities (LMIs).
\end{abstract}

AMS Subject Classification: 92B05, 93D20, 37C75

Key Words: neural networks, guaranteed cost control, stabilization, multiple time-varying delays, Lyapunov function, linear matrix inequalities

\section{Introduction}

There has been great interest recently in dynamical characteristics of neural

Received: June 2, 2014

(c) 2014 Academic Publications, Ltd. url: www.acadpubl.eu 
networks or neural nets since Hopfield constructed a simplified neural network model, in which each neuron [1-8] is represented by a linear circuit consisting of a resistor and a capacitor, and is connected to other neurons via nonlinear sigmoidal activation functions, called transfer functions. Based on the Hopfield neural network model, Marcus and Westervelt argued that the nonlinear sigmoidal activation functions which connected to the other neurons would include discrete delays.

Guaranteed cost control problem [9-12] has the advantage of providing an upper bound on a given system performance index and thus the system performance degradation incurred by the uncertainties or time delays is guaranteed to be less than this bound. Nevertheless, despite such diversity of results available, most existing work either assumed that the time delays are constant or differentiable $[13,14]$. Although, in some cases, delay-dependent guaranteed cost control for systems with time-varying delays were considered in [12-14], the approach used there can not be applied to systems with interval, non-differentiable time-varying delays.

In this paper, we investigate the guaranteed cost control for Hopfield delayed neural networks problem with multiple time-varying delays. The novel features here are that the delayed neural network under consideration is with various globally Lipschitz continuous activation functions, and the multiple time-varying delays function is interval, non-differentiable. A nonlinear cost function is considered as a performance measure for the closed-loop system. The stabilizing controllers to be designed must satisfy some exponential stability constraints on the closed-loop poles. Based on constructing a set of augmented Lyapunov-Krasovskii functional combined with Newton-Leibniz formula, new delay-dependent criteria for guaranteed cost control via memoryless feedback control is established in terms of LMIs.

The outline of the paper is as follows. Section 2 presents definitions and some well-known technical propositions needed for the proof of the main result. LMI delay-dependent criteria for guaranteed cost control is presented in Section 3. The paper ends with conclusions and cited references.

\section{Preliminaries}

The following notation will be used in this paper. $\mathbb{R}^{+}$denotes the set of all real non-negative numbers; $\mathbb{R}^{n}$ denotes the $n$-dimensional space with the scalar product $\langle x, y\rangle$ or $x^{T} y$ of two vectors $x, y$, and the vector norm $\|$. $\|$; $M^{n \times r}$ denotes the space of all matrices of $(n \times r)$-dimensions. $A^{T}$ denotes the 
transpose of matrix $A ; A$ is symmetric if $A=A^{T} ; I$ denotes the identity matrix; $\lambda(A)$ denotes the set of all eigenvalues of $A ; \lambda_{\max }(A)=\max \{\operatorname{Re} \lambda ; \lambda \in \lambda(A)\}$. $x_{t}:=\{x(t+s): s \in[-h, 0]\},\left\|x_{t}\right\|=\sup _{s \in[-h, 0]}\|x(t+s)\| ; C^{1}\left([0, t], \mathbb{R}^{n}\right)$ denotes the set of all $\mathbb{R}^{n}$-valued continuously differentiable functions on $[0, t]$; $L_{2}\left([0, t], \mathbb{R}^{m}\right)$ denotes the set of all the $\mathbb{R}^{m}$-valued square integrable functions on $[0, t]$;

Matrix $A$ is called semi-positive definite $(A \geq 0)$ if $\langle A x, x\rangle \geq 0$, for all $x \in \mathbb{R}^{n} ; A$ is positive definite $(A>0)$ if $\langle A x, x\rangle>0$ for all $x \neq 0 ; A>B$ means $A-B>0$. The notation $\operatorname{diag}\{\ldots\}$ stands for a block-diagonal matrix. The symmetric term in a matrix is denoted by $*$.

Consider the following Hopfield neural networks with interval multiple timevarying delays:

$$
\begin{aligned}
& \dot{x}(t)=-A x(t)+W_{0} f(x(t))+\sum_{i=1}^{m} W_{1 i} g\left(x\left(t-h_{i}(t)\right)\right)+B u(t), \quad t \geq 0, \\
& i=1,2, \ldots, m, \quad x(t)=\phi(t), t \in\left[-h_{1 i}, 0\right], \quad i=1,2, \ldots, m,
\end{aligned}
$$

where $x(t)=\left[x_{1}(t), x_{2}(t), \ldots, x_{n}(t)\right]^{T} \in \mathbb{R}^{n}$ is the state of the neural, $u(.) \in$ $L_{2}\left([0, t], \mathbb{R}^{m}\right)$ is the control; $n$ is the number of neurals, and

$$
\begin{aligned}
& f(x(t))=\left[f_{1}\left(x_{1}(t)\right), f_{2}\left(x_{2}(t)\right), \ldots, f_{n}\left(x_{n}(t)\right)\right]^{T}, \\
& g(x(t))=\left[g_{1}\left(x_{1}(t)\right), g_{2}\left(x_{2}(t)\right), \ldots, g_{n}\left(x_{n}(t)\right)\right]^{T},
\end{aligned}
$$

are the activation functions; $A=\operatorname{diag}\left(\bar{a}_{1}, \bar{a}_{2}, \ldots, \bar{a}_{n}\right), \bar{a}_{i}>0$ represents the self-feedback term; $B \in R^{n \times m}$ is control input matrix; $W_{0}, W_{1}$ denote the connection weights, the discretely delayed connection weights and the distributively delayed connection weight, respectively; The multiple time-varying delays function $h_{i}(t), i=1,2, \ldots, m$ satisfies the condition

$$
0 \leq h_{0 i} \leq h_{i}(t) \leq h_{1 i}, \quad i=1,2, \ldots, m,
$$

The initial functions $\phi(t) \in C^{1}\left(\left[-h_{1 i}, 0\right], R^{n}\right)$, with the norm

$$
\|\phi\|=\sup _{t \in\left[-h_{1 i}, 0\right]} \sqrt{\|\phi(t)\|^{2}+\|\dot{\phi}(t)\|^{2}} .
$$

In this paper we consider various activation functions and assume that the activation functions $f(),. g($.$) are Lipschitzian with the Lipschitz constants f_{i}, e_{i}>$ 0 :

$$
\begin{aligned}
& \left|f_{i}\left(\xi_{1}\right)-f_{i}\left(\xi_{2}\right)\right| \leq f_{i}\left|\xi_{1}-\xi_{2}\right|, \quad i=1,2, \ldots, n, \forall \xi_{1}, \xi_{2} \in \mathbb{R} \\
& \left|g_{i}\left(\xi_{1}\right)-g_{i}\left(\xi_{2}\right)\right| \leq e_{i}\left|\xi_{1}-\xi_{2}\right|, \quad i=1,2, \ldots, n, \forall \xi_{1}, \xi_{2} \in \mathbb{R}
\end{aligned}
$$


The performance index associate with the system (1) is the following function

$$
J=\int_{0}^{\infty} f^{0}(t, x(t), x(t-h(t)), u(t)) d t
$$

where $f^{0}(t, x(t), x(t-h(t)), u(t)): R^{+} \times R^{n} \times R^{n} \times R^{m} \rightarrow R^{+}$, is a nonlinear cost function satisfies

$$
\exists Q_{1}, Q_{2}, R: f^{0}(t, x, y, u) \leq\left\langle Q_{1} x, x\right\rangle+\left\langle Q_{2} y, y\right\rangle+\langle R u, u\rangle,
$$

for all $(t, x, u) \in R^{+} \times R^{n} \times R^{m}$ and $Q_{1}, Q_{2} \in R^{n \times n}, R \in R^{m \times m}$, are given symmetric positive definite matrices. The objective of this paper is to design a memoryless state feedback controller $u(t)=K x(t)$ for system (1) and the cost function (3) such that the resulting closed-loop system

$$
\dot{x}(t)=(A+B K) x(t)+W_{0} f(x(t))+\sum_{i=1}^{m} W_{1 i} g\left(x\left(t-h_{i}(t)\right)\right), \quad i=1,2, \ldots, m,
$$

is exponentially stable and the closed-loop value of the cost function (3) is minimized.

Definition 1. Given $\alpha>0$. The zero solution of closed-loop system (5) is $\alpha$-exponentially stabilizable if there exist a positive number $N>0$ such that every solution $x(t, \phi)$ satisfies the following condition:

$$
\|x(t, \phi)\| \leq N e^{-\alpha t}\|\phi\|, \quad \forall t \geq 0 .
$$

Definition 2. Consider the control system (1). If there exist a memoryless state feedback control law $u^{*}(t)=K x(t)$ and a positive number $J^{*}$ such that the zero solution of the closed-loop system (5) is exponentially stable and the cost function (3) satisfies $J \leq J^{*}$, then the value $J^{*}$ is a guaranteed costant and $u^{*}(t)$ is a guaranteed cost control law of the system and its corresponding cost function.

We introduce the following technical well-known propositions, which will be used in the proof of our results.

Proposition 1.(Schur complement lemma [15]). Given constant matrices $X, Y, Z$ with appropriate dimensions satisfying $X=X^{T}, Y=Y^{T}>0$. Then $X+Z^{T} Y^{-1} Z<0$ if and only if

$$
\left(\begin{array}{cc}
X & Z^{T} \\
Z & -Y
\end{array}\right)<0
$$


Proposition 2. (Integral matrix inequality [15]). For any symmetric positive definite matrix $M>0$, scalar $\gamma>0$ and vector function $\omega:[0, \gamma] \rightarrow \mathbb{R}^{n}$ such that the integrations concerned are well defined, the following inequality holds

$$
\left(\int_{0}^{\gamma} \omega(s) d s\right)^{T} M\left(\int_{0}^{\gamma} \omega(s) d s\right) \leq \gamma\left(\int_{0}^{\gamma} \omega^{T}(s) M \omega(s) d s\right)
$$

\section{Main Results}

In this section, we give a design of memoryless guaranteed feedback cost control for neural networks (1). Let us set

$$
\begin{aligned}
& W_{11 i}=-[P+\alpha I] A-A^{T}[P+\alpha I]-2 B B^{T}+0.25 B R B^{T}+\sum_{i=0}^{1} G_{i}, \\
& W_{12 i}=P+A P+0.5 B B^{T}, W_{13 i}=e^{-2 \alpha h_{0}} H_{0}+0.5 B B^{T}+A P \\
& W_{14 i}=2 e^{-2 \alpha h_{1 i}} H_{1}+0.5 B B^{T}+A P, W_{15 i}=P 0.5 B B^{T}+A P, \\
& W_{22 i}=W_{0} D_{0} W_{0}^{T}+\sum_{i=1}^{m} W_{1 i} D_{1 i} W_{1 i}^{T}+\sum_{i=0}^{1} h_{i}^{2} H_{i}+\left(h_{1}-h_{0}\right) U-2 P-B B^{T}, \\
& W_{23 i}=P, \quad W_{24 i}=P, \quad W_{25 i}=P, \\
& W_{33 i}=-e^{-2 \alpha h_{0 i}} G_{0}-e^{-2 \alpha h_{0 i}} H_{0}-e^{-2 \alpha h_{1 i}} U+W_{0} D_{0} W_{0}^{T}+\sum_{i=1}^{m} W_{1 i} D_{1 i} W_{1 i}^{T}, \\
& W_{34 i}=0, \quad W_{35 i}=-2 \alpha h_{1 i} U, \\
& W_{44 i}=W_{0} D_{0} W_{0}^{T}+\sum_{i=1}^{m} W_{1 i} D_{1 i} W_{1 i}^{T}-e^{-2 \alpha h_{1 i}} U-e^{-2 \alpha h_{1 i}} G_{1}-e^{-2 \alpha h_{1 i}} H_{1}, \\
& W_{45 i}=e^{-2 \alpha h_{1 i}} U, W_{55 i}=-e^{-2 \alpha h_{1 i}} U+W_{0} D_{0} W_{0}^{T}, \\
& E=\operatorname{diag}\left\{e_{i}, i=1, \ldots, n\right\}, \quad F=\operatorname{diag}\left\{f_{i}, i=1, \ldots, n\right\}, \lambda_{1}=\lambda_{\min }\left(P^{-1}\right), \\
& \lambda_{2}=\lambda_{\max }\left(P^{-1}\right)+h_{0 i} \lambda_{\max }\left[P^{-1}\left(\sum_{i=0}^{1} G_{i}\right) P^{-1}\right] \\
& E_{i=0}\left[P_{i}^{-1}\left(H_{i}\right) P^{-1}\right]+\left(h_{1 i}-h_{0 i}\right) \lambda_{\max }\left(P^{-1} U P^{-1}\right) .
\end{aligned}
$$


Theorem 3.1. Consider control system (1) and the cost function (3). If there exist symmetric positive definite matrices $P, U, G_{0}, G_{1}, H_{0}, H_{1}$, and diagonal positive definite matrices $D_{0}, D_{1 i}, i=1,2, \ldots, m$ satisfying the following LMIS

$$
\begin{aligned}
& {\left[\begin{array}{ccccl}
W_{11 i} & W_{12 i} & W_{13 i} & W_{14 i} & W_{15 i} \\
* & W_{22 i} & W_{23 i} & W_{24 i} & W_{25 i} \\
* & * & W_{33 i} & W_{34 i} & W_{35 i} \\
* & * & * & W_{44 i} & W_{45 i} \\
* & * & * & * & W_{55 i}
\end{array}\right]<0, \quad i=1,2, \ldots, m} \\
& {\left[\begin{array}{ccc}
-P A-A^{T} P-e^{-2 \alpha h_{0 i}} H_{0}-e^{-2 \alpha h_{1 i}} H_{1} & 2 P F & P Q_{1} \\
* & -D_{0} & 0 \\
* & * & -Q_{1}^{-1}
\end{array}\right]<0, \quad i=1,2, \ldots, m,} \\
& {\left[\begin{array}{ccc}
W_{1 i} D_{1 i} W_{1 i}^{T}-e^{-2 \alpha h_{1 i} U} & 2 P E & P Q_{2} \\
* & -D_{1 i} & 0 \\
* & * & -Q_{2}^{-1}
\end{array}\right]<0, \quad i=1,2, \ldots, m}
\end{aligned}
$$

then

$$
u(t)=-\frac{1}{2} B^{T} P^{-1} x(t), \quad t \geq 0 .
$$

is a guaranteed cost control and the guaranteed cost value is given by

$$
J^{*}=\lambda_{2}\|\phi\|^{2}
$$

Moreover, the solution $x(t, \phi)$ of the system satisfies

$$
\|x(t, \phi)\| \leq \sqrt{\frac{\lambda_{1}}{\lambda_{2}}} e^{-\alpha t}\|\phi\|, \quad \forall t \geq 0 .
$$

Proof. Let $Y=P^{-1}, y(t)=Y x(t)$. Using the feddback control (2.5) we consider the following Lyapunov-Krasovskii functional

$$
V\left(t, x_{t}\right)=\sum_{i=1}^{6} V_{i}\left(t, x_{t}\right)
$$




$$
\begin{aligned}
& V_{1}=x^{T}(t) Y x(t), \\
& V_{2}=\int_{t-h_{0}}^{t} e^{2 \alpha(s-t)} x^{T}(s) Y G_{0} Y x(s) d s, \\
& V_{3}=\int_{t-h_{1}}^{t} e^{2 \alpha(s-t)} x^{T}(s) Y G_{1} Y x(s) d s, \\
& V_{4}=h_{0} \int_{-h_{0}}^{0} \int_{t+s}^{t} e^{2 \alpha(\tau-t)} \dot{x}^{T}(\tau) Y H_{0} Y \dot{x}(\tau) d \tau d s, \\
& V_{5}=h_{1} \int_{-h_{1}}^{0} \int_{t+s}^{t} e^{2 \alpha(\tau-t)} \dot{x}^{T}(\tau) Y H_{1} Y \dot{x}(\tau) d \tau d s, \\
& V_{6}=\left(h_{1}-h_{0}\right) \int_{t-h_{1}}^{t-h_{0}} \int_{t+s}^{t} e^{2 \alpha(\tau-t)} \dot{x}^{T}(\tau) Y U Y \dot{x}(\tau) d \tau d s .
\end{aligned}
$$

It easy to check that

$$
\lambda_{1}\|x(t)\|^{2} \leq V\left(t, x_{t}\right) \leq \lambda_{2}\left\|x_{t}\right\|^{2}, \quad \forall t \geq 0
$$

Taking the derivative of $V_{i}, i=1,2, \ldots, 6$, we have

$$
\begin{gathered}
\dot{V}_{1}=2 x^{T}(t) Y \dot{x}(t) \\
=y^{T}(t)\left[-P A^{T}-A P\right] y(t)-y^{T}(t) B B^{T} y(t) \\
\quad+2 y^{T}(t) W_{0} f(.) y(t)+2 y^{T}(t) \sum_{i=1}^{m} W_{1 i} g\left(x\left(t-h_{i}(t)\right)\right) y(t) \\
\dot{V}_{2}=y^{T}(t) G_{0} y(t)-e^{-2 \alpha h_{0}} y^{T}\left(t-h_{0}\right) G_{0} y\left(t-h_{0}\right)-2 \alpha V_{2} ; \\
\dot{V}_{3}=y^{T}(t) G_{1} y(t)-e^{-2 \alpha h_{1}} y^{T}\left(t-h_{1}\right) G_{1} y\left(t-h_{1}\right)-2 \alpha V_{3} ; \\
\dot{V}_{4}=h_{0}^{2} \dot{y}^{T}(t) H_{0} \dot{y}(t)-h_{1} e^{-2 \alpha h_{0}} \int_{t-h_{0}}^{t} \dot{x}^{T}(s) H_{0} \dot{x}(s) d s-2 \alpha V_{4} ; \\
\dot{V}_{5}=h_{1}^{2} \dot{y}^{T}(t) H_{1} \dot{y}(t)-h_{1} e^{-2 \alpha h_{1}} \int_{t-h_{1}}^{t} \dot{y}^{T}(s) H_{1} \dot{y}(s) d s-2 \alpha V_{4} ; \\
\dot{V}_{6}=\left(h_{1}-h_{0}\right)^{2} \dot{y}^{T}(t) U \dot{y}(t)-\left(h_{1}-h_{0}\right) e^{-2 \alpha h_{1}} \int_{t-h_{1}}^{t-h_{0}} \dot{y}^{T}(s) U \dot{y}(s) d s-2 \alpha V_{6} .
\end{gathered}
$$

we obtain

$$
\begin{gathered}
\dot{V}(.)+2 \alpha V(.) \leq \zeta^{T}(t) \mathcal{E}_{i} \zeta(t)+y^{T}(t) S_{1 i} y(t)+y^{T}\left(t-h_{i}(t)\right) S_{2 i} y\left(t-h_{i}(t)\right) \\
-f^{0}\left(t, x(t), x\left(t-h_{i}(t)\right), u(t)\right)
\end{gathered}
$$


where $\zeta(t)=\left[y(t), \dot{y}(t), y\left(t-h_{0 i}\right), y\left(t-h_{1 i}\right), y\left(t-h_{i}(t)\right), f(x(t)), g\left(x\left(t-h_{i}(t)\right)\right)\right]$, and

$$
\begin{gathered}
\mathcal{E}_{i}=\left[\begin{array}{ccccc}
W_{11 i} & W_{12 i} & W_{13 i} & W_{14 i} & W_{15 i} \\
* & W_{22 i} & W_{23 i} & W_{24 i} & W_{25 i} \\
* & * & W_{33 i} & W_{34 i} & W_{35 i} \\
* & * & * & W_{44 i} & W_{45 i} \\
* & * & * & * & W_{55 i}
\end{array}\right], \quad i=1,2, \ldots, m, \\
S_{1 i}=-P A-A^{T} P-e^{-2 \alpha h_{0 i}} H_{0}-e^{-2 \alpha h_{1 i}} H_{1}+4 P F D_{0}^{-1} F P+P Q_{1} P, \quad i=1,2, \ldots, m, \\
S_{2 i}=W_{1 i} D_{1 i} W_{1 i}^{T}-e^{-2 \alpha h_{2 i}} U+4 P E D_{1 i}^{-1} E P+P Q_{2} P, \quad i=1,2, \ldots, m .
\end{gathered}
$$

Note that by the Schur complement lemma, Proposition 2.1, the conditions $S_{1 i}<0$ and $S_{2 i}<0$ are equivalent to the conditions (7) and (8), respectively. Therefore, by condition (6), (7), (8), we obtain from (14) that

$$
\dot{V}\left(t, x_{t}\right) \leq-2 \alpha V\left(t, x_{t}\right), \quad \forall t \geq 0 .
$$

Integrating both sides of (15) from 0 to $t$, we obtain

$$
V\left(t, x_{t}\right) \leq V(\phi) e^{-2 \alpha t}, \quad \forall t \geq 0 .
$$

Furthermore, taking condition (3.5) into account, we have

$$
\lambda_{1}\|x(t, \phi)\|^{2} \leq V\left(x_{t}\right) \leq V(\phi) e^{-2 \alpha t} \leq \lambda_{2} e^{-2 \alpha t}\|\phi\|^{2},
$$

then

$$
\|x(t, \phi)\| \leq \sqrt{\frac{\lambda_{2}}{\lambda_{1}}} e^{-\alpha t}\|\phi\|, \quad t \geq 0,
$$

which concludes the exponential stability of the closed-loop system (5). To prove the optimal level of the cost function (3), we derive from (14) and (6) (8) that

$$
\dot{V}\left(t, z_{t}\right) \leq-f^{0}\left(t, x(t), x\left(t-h_{i}(t)\right), u(t)\right), \quad t \geq 0 .
$$

Integrating both sides of (16) from 0 to $t$ leads to

$$
\int_{0}^{t} f^{0}\left(t, x(t), x\left(t-h_{i}(t)\right), u(t)\right) d t \leq V\left(0, z_{0}\right)-V\left(t, z_{t}\right) \leq V\left(0, z_{0}\right),
$$

dute to $V\left(t, z_{t}\right) \geq 0$. Hence, letting $t \rightarrow+\infty$, we have

$$
J=\int_{0}^{\infty} f^{0}\left(t, x(t), x\left(t-h_{i}(t)\right), u(t)\right) d t \leq V\left(0, z_{0}\right) \leq \lambda_{2}\|\phi\|^{2}=J^{*} .
$$

This completes the proof of the theorem. 


\section{Conclusion}

In this paper, the problem of guaranteed cost control for Hopfield neural networks with interval multiple nondifferentiable time-varying delays has been studied. A nonlinear quadratic cost function is considered as a performance measure for the closed-loop system. The stabilizing controllers to be designed must satisfy some exponential stability constraints on the closed-loop poles.

\section{Acknowledgments}

This work was supported by the Thailand Research Fund Grant, the Commission for Higher Education and Faculty of Science, Maejo University, Thailand. The authors thank anonymous reviewers for valuable comments and suggestions, which allowed us to improve the paper.

\section{References}

[1] J.J. Hopfield, Neural networks and physical systems with emergent collective computational abilities, Proc. Natl. Acad. Sci. USA, 79(1982), 2554-2558.

[2] VN. Phat, Y. Kongtham, and K. Ratchagit, LMI approach to exponential stability of linear systems with interval time-varying delays, Linear Algebra Appl., Vol. 436, pp. 243-251, 2012. doi: 10.1016/j.laa.2011.07.016

[3] G. Rajchakit, Delay-dependent optimal guaranteed cost control of stochastic neural networks with interval nondifferentiable time-varying delays, ADVANCES IN DIFFERENCE EQUATIONS, 2013, 1-11. DOI: 10.1186/1687-1847-2013-241

[4] K. Ratchagit, A switching rule for the asymptotic stability of discrete-time systems with convex polytopic uncertainties, Asian-European Journal of Mathematics, Vol. 5, 2012. DOI: 10.1142/S1793557112500258

[5] Kreangkri Ratchagit, STABILITY ANALYSIS OF LINEAR SYSTEMS WITH TIME DELAYS, International Journal of Pure and Applied Mathematics, Vol. 76 No. 1, 2012, 21-28. 
[6] K. Ratchagit, Asymptotic stability of delay-difference system of Hopfield neural networks via matrix inequalities and application, International Journal of Neural Systems, 17(2007), 425-430. DOI: 10.1142/S0129065707001263

[7] K. Ratchagit, V.N. Phat, Stability and stabilization of switched linear discrete-time systems with interval time-varying delay, Nonlinear Anal. Hybrid Syst. 5 (2011) 605-612. DOI: 10.1016/j.nahs.2011.05.006

[8] K. Ratchagit , THE SUFFICIENT CONDITIONS FOR STABILITY OF LINEAR TIME-VARYING SYSTEMS WITH STATE DELAYS, International Journal of Pure and Applied Mathematics, Vol. 65 No. 1, 2010, $65-72$.

[9] K. Ratchagit and V.N. Phat, Stability criterion for discrete-time systems, J. Ineq. Appl., 2010(2010), 1-6. doi:10.1155/2010/201459

[10] Grienggrai Rajchakit, Delay-Dependent Asymptotical Stabilization Criterion of Recurrent Neural Networks, Applied Mechanics and Materials. 330(2013) 1045-1048. doi:10.4028/www.scientific.net/AMM.330.1045

[11] K. Ratchagit, Asymptotic stability of nonlinear delay-difference system via matrix inequalities and application, International Journal of Computational Methods, pp. 389-397, 2009. DOI: 10.1142/S0219876209001899

[12] Kreangkri Ratchagit , EXPONENTIAL STABILITY OF SWITCHED LINEAR SYSTEMS, International Journal of Pure and Applied Mathematics, Vol. 58 No. 3, 2010, 361-371.

[13] P. Niamsup, G. Rajchakit, New Results on Robust Stability and Stabilization of Linear Discrete-Time Stochastic Systems with Convex Polytopic Uncertainties, JOURNAL OF APPLIED MATHEMATICS, 2013(2013). DOI: $10.1155 / 2013 / 368259$

[14] G. Rajchakit, Stabilization of switched discrete-time systems with convex polytopic uncertainties, Journal of Computational Analysis and Applications 16 (2014) 20-29.

[15] Kreangkri Ratchagit, STABILITY CRITERIA OF LPD SYSTEM WITH TIME-VARYING DELAY, International Journal of Pure and Applied Mathematics, Vol. 78 No. 6, 2012, 857-866. 
[16] M. De la Sen and A. Ibeas, Stability Results of a Class of Hybrid Systems under Switched Continuous-Time and Discrete-Time Control, Discrete Dynamics in Nature and Society, 2009(2009).

[17] Manlika Rajchakit and Grienggrai Rajchakit, Mean Square Exponential Stability of Stochastic Switched System with Interval Time-Varying Delays, Abstract and Applied Analysis, vol. 2012, Article ID 623014, 12 pages, 2012. doi: $10.1155 / 2012 / 623014$

[18] M Rajchakit, G Rajchakit, Mean square robust stability of stochastic switched discrete-time systems with convex polytopic uncertainties, Journal of Inequalities and Applications 2012, 2012:135. doi:10.1186/1029242X-2012-135

[19] M. Rajchakit and G. Rajchakit, LMI approach to robust stability and stabilization of nonlinear uncertain discrete-time systems with convex polytopic uncertainties, Advances in Difference Equations, 2012(2012). doi:10.1186/1687-1847-2012-106

[20] R.P. Agarwal, Difference Equations and Inequalities, Second Edition, Marcel Dekker, New York, 2000. 
